\title{
INTERAÇÕES COMPORTAMENTAIS EM ESQUEMA MÚLTIPLO UTILIZANDO O JATO DE AR QUENTE (JAQ) COMO ESTÍMULO PUNIDOR
}

\author{
BEHAVIORAL INTERACTIONS IN MULTIPLE SCHEDULE USING HOT BLAST AIR (HBA) AS PUNISHER \\ STIMULUS
}

BRUNA COLOMBO DOS SANTOS

HELoÍSA QUARESMA PUREZA

Marcus Bentes de CARVAlho Neto

UNIVERSIDAdE FEDERAL do PARÁ, BRASIL

\begin{abstract}
RESUMO
Interação comportamental é um fenômeno que pode ocorrer em esquemas múltiplos, em que a taxa de respostas em um componente pode mudar em função de alteração do esquema de reforçamento ou de punição no segundo componente. Interações comportamentais podem ocorrer quando há utilização de contingências apetitivas ou aversivas, como punição. Com relação às contingências punitivas, existem dados conflitantes na literatura sobre a produção de contraste ou indução com não humanos, utilizando majoritariamente choque elétrico como estímulo punidor. O objetivo deste estudo foi verificar se ocorreria algum tipo de interação comportamental em esquemas múltiplos, utilizando o jato de ar quente (JAQ) como estímulo punidor. Dezesseis ratos albinos foram expostos às fases: Pré-Punição, Punição e Pós-Punição. Nas três fases vigorou um esquema múltiplo com dois componentes (claro e escuro). O esquema de reforçamento em vigor em ambos os componentes foi intervalo variável 30s (VI 30) para respostas de pressão à barra, em todas as fases. Na fase punição, o JAQ foi administrado contingente a cada resposta de pressão à barra no componente claro para metade dos sujeitos, e no escuro para a outra metade. Ocorreu indução negativa no componente não punido, para todos os sujeitos, principalmente nas sessões iniciais; ao longo das sessões, ocorreu recuperação na taxa nesse componente para todos os sujeitos, resultando em ocorrência tardia de contraste positivo para três dos sujeitos. A produção de contraste parece estar relacionada ao número de sessões de exposição ao punidor.
\end{abstract}

Palavras-chave: interação comportamental, punição, contraste, indução.

\begin{abstract}
Behavioral interaction is a phenomenon that can occur in multiple schedules, in which the response rate in one component can change due to alterations in reinforcement or punishment schedules, in another component. Behavioral interactions can occur under appetitive or aversive contingencies, such as punishment contingencies. Regarding punishment contingencies, the literature reports conflicting data about the occurrence of contrast or induction with non-humans, mostly when electric shock is the punisher. The goal of this study was to assess the occurrence of behavioral interactions in multiple schedules using the hot blast air (HBA) as punisher. Sixteen albino rats were exposed to the phases: Pre-Punishment, Punishment, and Post-Punishment. In all three phases, a multiple schedule with two components was in effect. The reinforcement schedule for bar press responding in both components was variable interval 30-s (VI 30 s), in all phases. In Punishment phase, the HBA was administered contingent on each bar pressing response, in the light component for half of the subjects, and in the dark for the other half. Negative induction occurred in the non-punishment component, mainly in the initial punishment sessions; response rates in this component recovered across sessions, and, for three rats, resulted in behavioral contrast. The occurrence of contrast seems to be related to the number of sessions of exposure to the punisher.
\end{abstract}

Keywords: behavioral interaction, punishment, contrast, induction.

Endereço para correspondência: Rua Augusto Corrêa, 01, Campos Universitário do Guamá, Belém, Pará, Brasil, CEP: 66075110. Telefone: (91) 3201-8542/8476. Email: brucolombodossantos@gmail.com. Trabalho financiado pela CAPES por meio de bolsas de doutorado (1 ${ }^{\text {a }}$ autora) e mestrado ( $2^{\mathrm{a}}$ autora) e do CNPq, por meio de bolsa de produtividade em pesquisa ( $3^{\circ}$ autor) e de auxílio no Edital Universal (Processo No. 476839/2013-0). 
Em esquemas múltiplos, a taxa de respostas em um componente, na presença de determinado estímulo, pode mudar em função de alteração do esquema de reforçamento em outro componente, associado com outro estímulo. Esse fenômeno é chamado de interação comportamental (Herrnstein \& Brady, 1958; Reynolds, 1961a).

A interação comportamental é dividida em dois tipos: (1) contraste e (2) indução. Contraste ocorre quando há uma mudança na taxa de respostas em um componente do esquema múltiplo (componente estável) que vai na direção oposta à da mudança na taxa de respostas no componente cujo esquema de reforçamento varia. Indução ocorre quando a taxa de respostas no componente estável muda na mesma direção da taxa de respostas no componente variável (Reynolds, 1961a).

Esses dois tipos, por sua vez, são classificados em (1) positivo e (2) negativo. Sendo assim, quatro tipos de interações comportamentais são possíveis: (1) contraste positivo - ocorre quando há um aumento na taxa de respostas no componente estável em função da diminuição da taxa de respostas no componente alterado; (2) contraste negativo - ocorre quando há diminuição na taxa de respostas no componente estável em função do aumento na taxa de respostas no componente alterado; (3) indução positiva ocorre quando há um aumento na taxa de respostas no componente estável em função do aumento na taxa no componente alterado; (2) indução negativa - ocorre quando há diminuição na taxa de respostas no componente estável em função da diminuição na taxa no componente alterado (Pear \& Wilkie, 1971; Reynolds, 1961b).

Estudos experimentais têm demonstrado a produção de interações comportamentais em contingências apetitivas, com diversos tipos de sujeitos (e.g., pombos, ratos, macacos), utilizando esquemas múltiplos (e.g., Bloomfield, 1967; Bradshaw, 1975; Caplan \& Graefe, 1980; Catania, 1961; Catania \& Gill, 1964; Hemmes \& Eckerman, 1972; Gutman, 1977; Gutman, Sutterer, \& Brush, 1975; MacDonall \& Marcucella, 1978; Pear \& Wilkie, 1971; Reynolds, 1961a, 1961b, 1961c; Reynolds \& Limpo, 1968; Spealman, 1978; Swindell, McSweeney, \& Murphy 2003; Thomas \& Cameron, 1974; Topping \& Larmi Jr, 1972). Interações comportamentais também foram demonstradas utilizando contingências aversivas - punição e reforçamento negativo (e.g., Azrin, 1956; Brethower \& Reynolds, 1962; Coates, 1972; Crosbie, Williams, Lattal, Anderson, \& Brown, 1997; Dinsmoor, 1952; Herrnstein \& Brady, 1958; Honig \& Slivka, 1964; Lattal, 1970; Lattal \& Griffin, 1972; Rachlin, 1966; Tullis \& Walters, 1968).

Todavia, com relação às contingências punitivas, existem dados conflitantes na literatura acerca da produção de interações comportamentais com não humanos. Há dados: (1) sobre produção apenas de contraste (Coates, 1972) ou apenas de indução (Dinsmoor, 1952); (2) sobre ocorrência tanto de indução quanto de contraste (Azrin \& Holz, 1966; Brethower \& Reynolds, 1962; Crosbie et al., 1997); ou (3) que são inconsistentes ou não demonstraram interação comportamental (Azrin, 1956; Lattal, 1970; Rachlin, 1966).

As variáveis que contribuem para a produção de indução, contraste ou ambos quando punição é utilizada, não são claras. Uma das variáveis importantes para a produção de contraste, por exemplo, é a taxa de reforçamento (Catania, 1961; Reynolds, 1961a; 1961b; Reynolds \& Limpo, 1968). Contraste ocorre quando há uma relação inversa entre a taxa de respostas no componente estável e a taxa de reforçamento no componente alterado (MacSweeney \& Norman, 1979).

Quando o esquema utilizado no componente alterado é punição, a taxa de respostas é suprimida e, portanto, há um efeito indireto sobre a frequência de reforçamento, pois o organismo não emite a resposta selecionada e a frequência de reforçamento cai. Esse efeito faz com que as duas variáveis covariem: (a) apresentação de um estímulo punidor e (b) diminuição na frequência de reforçamento (Crosbie et al., 1997).

Outras variáveis citadas são: (1) tempo de exposição ao estímulo punidor e intensidade desse estímulo (Brethower \& Reynolds, 1962); (2) diferenças de procedimento, como a utilização ou não de estímulos conspícuos associados a cada componente do esquema múltiplo (Gutman, Sutterer, \& Brush, 1975); (3) história prévia - número de exposições dos sujeitos ao estímulo punidor; (4) diferenças individuais - reatividade diferencial de cada organismo ao punidor; (5) maneira de introdução do estímulo punidor - abrupta ou gradualmente; e (6) fatores de estímulo - utilização do choque elétrico podendo mascarar os estímulos exteroceptivos programados em cada componente (Crosbie et al., 1997).

Nota-se que o choque elétrico foi o estímulo punidor majoritário utilizado em todas as pesquisas e, com exceção das considerações feitas por Brethower e Reynolds (1962) e Crosbie et al. (1997) a respeito desse estímulo, nenhum outro estudo investigou ou considerou se esse tipo de estímulo punidor poderia influenciar na produção ou não de interações comportamentais ou no tipo de interação produzida, com não humanos.

Ademais, o uso majoritário do choque elétrico tem sido apontando como um dos motivos pelos quais as pesquisas em controle aversivo têm declinado em comparação com outras temáticas de pesquisa em Análise do Comportamento (Baron, 1991; Catania, 2008). Segundo Catania (2008), as implicações éticas e ecológicas desse estímulo parecem ter comprometido o avanço da pesquisa nessa área e, também, a generalidade dos dados obtidos.

Por essa razão, a busca por formas de estimulação aversiva alternativas ao choque elétrico parece crucial para uma retomada das pesquisas sobre controle aversivo e para testar a generalidade das leis comportamentais estabelecidas com tal estímulo (Hurtado-Parrado, 2015). Por exemplo, alguns fenômenos que envolvem contingências aversivas como aversão gustativa, apresentam diferenças significativas nos dados, a depender do tipo de estímulo aversivo utilizado 
(Hoffman, Hunt, \& Spear, 1991; Pelchat, Grill, Rozin, \& Jacobs, 1983) e, recentemente, Hurtado-Parrado (2015) não produziu respostas de esquiva em peixes (betta splendens) utilizando fluxo de água como estímulo aversivo. Dessa forma, o teste de novos estímulos, diferentes do choque elétrico, parece crucial para a produção de novos dados sobre controle aversivo, que sejam mais consistentes e generalizáveis, dentre eles dados sobre interações comportamentais.

Nessa linha, o jato de ar quente (JAQ) tem se mostrado um estímulo aversivo eficaz alternativo ao choque elétrico em: (a) condições de contingência e não contingência (Carvalho Neto, Neves Filho, Borges, \& Tobias, 2007; Carvalho Neto, Rico, Tobias, Gouveia Jr, \& Angerami, 2005); (b) esquemas contínuos e intermitentes de punição (Carvalho Neto, Maestri, \& Menezes, 2007; Carvalho Neto et al, 2005); (c) procedimento de supressão condicionada (Nascimento \& Carvalho Neto, 2011); (d) procedimentos de discriminação simples (Carvalho Neto, Costa, Barros, Farias, \& Rico, 2013) e (e) sobre respostas instrumentais - que permitem acesso a uma consequência específica - e consumatórias - que são respostas relacionadas à consequência em si, como mastigar, lamber ou engolir (Mayer, Silva, \& Carvalho Neto, 2015).

Observando os dados conflitantes sobre a produção de contraste ou indução em esquemas múltiplos com punição, o uso majoritário de choque com não humanos e a possibilidade de mais um teste sobre efetividade do JAQ como estímulo punidor, o objetivo deste estudo foi verificar se há produção de algum tipo de interação comportamental e, se sim, qual tipo (indução ou contraste), utilizando o JAQ como estímulo punidor e ratos como sujeitos, em um esquema múltiplo de reforçamento positivo com dois componentes.

Este trabalho contribui diretamente para o aumento na generalidade dos dados produzidos com o uso do JAQ, para a verificação da generalidade de um processo comportamental básico, quando outro tipo de estímulo é utilizado e, mais especificamente, para a discussão sobre tipos de interações comportamentais que ocorrem quando punição é utilizada e possíveis variáveis que influenciam em sua produção.

\section{Sujeitos}

\section{MÉTODO}

Foram utilizados 16 ratos albinos (Rattus norvegicus, Wistar), machos, experimentalmente ingênuos, com aproximadamente doze semanas de vida no início do experimento e provenientes do Biotério do Instituto Evandro Chagas (IEC). Os sujeitos foram mantidos no Biotério do Núcleo de Teoria e Pesquisa do Comportamento (NTPC; UFPA) em pares e ficaram privados de comida por aproximadamente 24 horas antes de cada sessão experimental, com água disponível continuamente nas gaiolas-viveiro. Após as sessões, $10 \mathrm{~g}$ de alimento eram disponibilizadas para cada animal, isoladamente, por uma hora na gaiola-viveiro. O estudo foi submetido e aprovado pelo Comitê de Ética de Pesquisa Com Animais (CEPAE) da Universidade Federal do Pará (UFPA), Protocolo Número 241-14.

\section{Equipamentos \& Materiais}

Foi utilizada uma caixa de condicionamento operante da MED Associates (mod. ENV-008-VP), com dimensões de 25,5 x 32,2 × $25 \mathrm{~cm}$ (comprimento, largura e altura), adaptada para o uso do JAQ: o piso de metal foi substituído por um piso de acrílico (barras de $0,5 \mathrm{~cm}$ ), uma barra de acrílico foi acoplada sobre a barra original de metal, o teto foi substituído por uma malha metálica vazada, e dois secadores de cabelo foram acoplados acima da malha metálica (para mais informações, ver Silva, Carvalho Neto, \& Mayer, 2014). O JAQ foi liberado pelos dois secadores de cabelo - marca REVLON, modelo RV429AB - que estavam conectados à torre de comando do equipamento para acionamento automatizado. O JAQ gerava, na intensidade adotada, um aumento de aproximadamente $2^{\circ} \mathrm{C}$ ao final de 5 segundos e um nível de ruído de $85 \mathrm{~dB}$ (para mais detalhes sobre as dimensões do JAQ, ver Rodrigues, Nascimento, Cavalcante, \& Carvalho Neto, 2008). Também havia três lâmpadas fluorescentes acopladas nas laterais da caixa, todas posicionadas acima da barra: duas na mesma lateral da barra, 7 cm acima da barra (L2 e L3), e uma na lateral oposta à lateral da barra, 9 centímetros acima da barra (L1). As lâmpadas eram acesas nas suas intensidades máximas (lâmpada de 127 V, 40 W, leitosa, modelo A/108/004, fabricada pela Sadokin) (Ver Figura 1). O comedouro, com dimensões 7,6 cm x $8,3 \mathrm{~cm}$, localizava-se logo abaixo da barra.

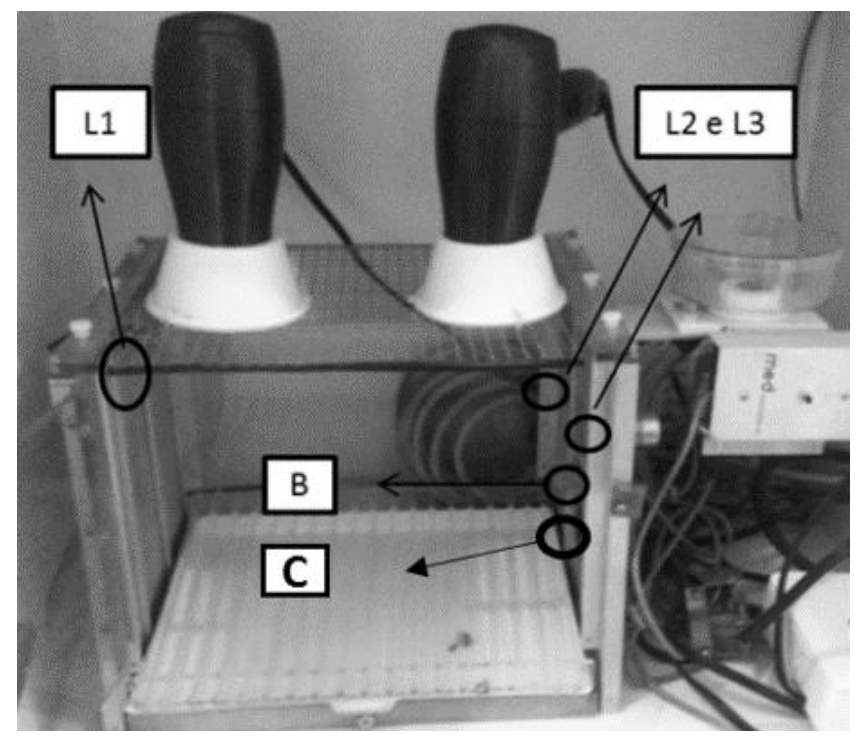

Figura 1. Caixa de condicionamento operante (MED Associates, mod. ENV-008-VP) adaptada para o uso do JAQ. As siglas L1, L2, L3, B e C correspondem, respectivamente, à: Luz 1, Luz 2, Luz 3, Barra e Comedouro. 
Foi utilizado um computador (Processador Intel ® Celeron ${ }^{\circledR}$, memória $1 \mathrm{~Gb}$ ) para controle automatizado do experimento e registro das respostas, por meio do programa MED-PC IV. Pelotas de alimento de $45 \mathrm{mg}$ (Dustless Precision Pellets) foram utilizadas como consequência para a resposta de pressão à barra.

\section{Procedimento}

Todos os sujeitos foram expostos às fases:

(1) Treino ao Comedouro e Modelagem da Resposta de Pressão à Barra. Ambos os procedimentos foram conduzidos em uma única sessão. Primeiro foi realizado o treino discriminativo do som da ativação do comedouro, cujo critério foi o sujeito se aproximar do comedouro assim que o som fosse liberado, independentemente de onde estivesse na câmara experimental. A partir disso, respostas de pressão à barra foram estabelecidas por reforçamento diferencial de aproximações sucessivas. $O$ critério de encerramento foi a duração da sessão (60 min).

(2) Fortalecimento da Resposta de Pressão à Barra (três sessões). Nessa fase os sujeitos foram expostos ao esquema razão fixa (FR1), em sessões de 60 minutos. As duas fases foram realizadas com todas as luzes apagadas para todos os sujeitos.

Os sujeitos foram então expostos aos esquemas múltiplos nas seguintes fases: Pré-Punição; Punição e PósPunição.

(3) Pré-Punição. Os sujeitos foram expostos a um esquema múltiplo com dois componentes, nos quais vigorava um esquema de intervalo variável (VI) 30s para a resposta de pressão à barra. O VI foi calculado utilizando a progressão de Fleshler e Hoffman (1962), tendo como número máximo 10 intervalos. Os intervalos eram selecionados após a emissão de uma resposta, ou seja, o VI ficava parado até que uma resposta fosse emitida para então selecionar o próximo intervalo. Não houve acúmulo de reforçadores entre os componentes (carryover). Os componentes diferiam quanto à presença ou ausência de luz: Componente Claro - todas as luzes acesas na intensidade máxima do equipamento; e Componente Escuro - todas as luzes apagadas. Os dois componentes (claro e escuro) se alternavam randomicamente; cada um ficava em vigor por 2 minutos, e não foi programado intervalo entre componentes.

(4) Punição. O esquema múltiplo VI 30s era mantido nos dois componentes, entretanto, cada resposta de pressão à barra também produziu um JAQ (punição contínua), em apenas um dos componentes. Os estímulos correlacionados com punição foram balanceados entre os sujeitos, isto é, oito sujeitos (PC1 a PC8) tiveram as respostas de pressão à barra consequenciadas com JAQ no componente claro; e oito sujeitos (PE1 a PE8) no componente escuro. Essa manipulação foi realizada para observar se os estímulos luz e escuro poderiam exercer alguma influência nos efeitos do
JAQ, pois a luz é demonstrada como um estímulo aversivo para ratos albinos (Keller, 1941; Keller,1966).

(5) Pós-Punição. A punição foi descontinuada e, portanto, essa fase foi idêntica à Pré-Punição.

Os sujeitos foram expostos a cada fase até que a taxa de respostas se estabilizasse por seis sessões consecutivas. O critério de estabilidade foi calculado com base em Schoenfeld, Cumming e Hearst (1956). Nesse critério, utiliza-se o último bloco de seis sessões e verifica-se se a diferença entre a média da taxa das três primeiras sessões e a média da taxa das três últimas é menor que 5\% da média da taxa de respostas do bloco inteiro (seis sessões). O cálculo da estabilidade foi realizado com o auxílio do software Stability Check (Costa \& Cançado, 2012). Todas as sessões tiveram duração de 60 minutos e eram realizadas em dias alternados, pois só era possível realizar sessão com um rato de cada vez, caso contrário o ruído do JAQ interferiria na sessão que estivesse ocorrendo concomitantemente. Como cada sessão durava uma hora e havia 16 sujeitos, não foi possível realizar sessões com todos os animais no mesmo dia. Em razão disso, optou-se por realizar as sessões de metade dos sujeitos em um dia e da outra metade no dia seguinte e assim sucessivamente.

\section{Análise de dados}

Foram analisadas as taxas de respostas por minuto em cada componente do esquema múltiplo, em todas as fases do experimento, bem como as taxas de reforços por minuto. Também foi analisada a diferença, em porcentagem, no total de respostas no componente não punido durante a fase Punição, em comparação com o total de respostas das três últimas sessões, nesse componente, na fase Pré-Punição (Brethower \& Reynolds, 1962). Para isso, foi feita a média do total de respostas no componente não punido nas três últimas sessões da fase Pré-Punição e o total de respostas no componente não punido, em cada sessão da fase Punição, foi divido por essa média e multiplicado por 100. Em seguida considerou-se o valor médio das três últimas sessões na fase Pré-Punição como representativo de $100 \%$, e esse valor foi subtraído das porcentagens obtidas em cada sessão da fase Punição, produzindo números negativos e/ou positivos. $\mathrm{O}$ número de sessões para que cada sujeito alcançasse o critério de estabilidade foi diferente; em razão disso as curvas apresentadas na Figura 4 terminam em momentos diferentes.

\section{RESULTADOS}

O objetivo deste estudo foi verificar a produção de interações comportamentais (contraste e/ou indução) utilizando o JAQ contingente à resposta de pressão à barra em um dos componentes (claro ou escuro) de um esquema múltiplo de intervalos variáveis (Mult VI 30s VI 30s).

As Figuras 2 e 3 apresentam os dados individuais com relação à taxa de respostas $(\mathrm{R} / \mathrm{min})$, de acordo com o balanceamento de sujeitos para os quais a punição foi introduzida, respectivamente, no componente claro ou no 
escuro. Observa-se um padrão semelhante nas curvas, para a maior parte dos sujeitos. Durante a Fase Pré-Punição, as taxas de respostas eram similares em ambos os componentes; quando o JAQ foi introduzido, observa-se que a taxa de respostas no componente punido foi suprimida e a taxa no componente estável também sofreu redução, mas em menor grau, seguindo a mudança de contingência do componente alterado (indução comportamental). Não houve diferença, com relação às mudanças na taxa de respostas, como função da administração do JAQ no componente claro ou escuro.
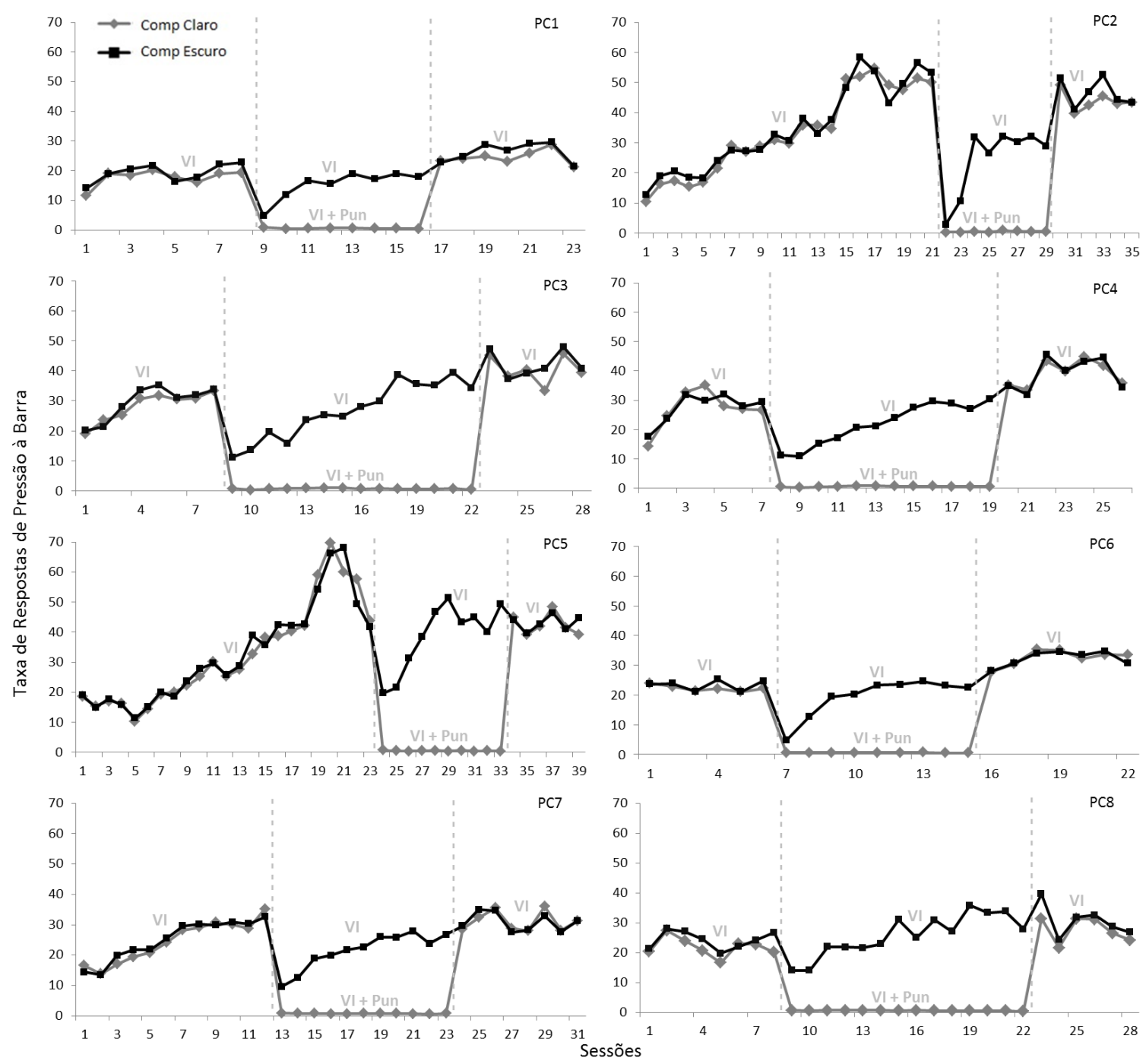

Figura 2. Taxa de respostas de pressão à barra em todas as sessões das fases Pré-Punição, Punição e Pós-Punição, quando o JAQ foi aplicado no componente claro (mudanças de fases representadas por linhas verticais tracejadas). A linha cinza representa a taxa de respostas no componente claro e a linha preta representa a taxa de resposta no componente escuro.

A diminuição na taxa de respostas no componente estável ocorreu de forma mais marcada na primeira sessão de introdução do JAQ no componente alterado. É importante destacar que a curva do sujeito PC5 apresentava, no final da fase Pré-Punição, tendência decrescente, o que pode ter contribuído para a queda na taxa observada na primeira sessão de punição. Após a primeira sessão de introdução do JAQ, a taxa de respostas começou a se recuperar no componente estável, entretanto, para a maioria dos sujeitos, não ultrapassou as taxas obtidas durante a fase Pré-Punição.
Foram exceções os sujeitos PC3, PC8 e PE1, cuja taxa de respostas no componente estável ultrapassou, durante a fase Punição, a taxa de respostas desse mesmo componente na fase Pré-Punição. Porém, o aumento na taxa ocorreu nas fases finais da punição: para o sujeito PC3 isso ocorreu na sessão 18, para PC8 nas sessões 15, 17, 19, 20 e 21; e para PE1 a partir da sessão 17. Sendo assim, observa-se que ocorreu indução negativa para todos os sujeitos, principalmente nas sessões iniciais; ao longo das sessões, 
ocorreu recuperação na taxa para todos os sujeitos, resultando em contraste positivo para três dos sujeitos.

Quando o JAQ foi retirado, as taxas no componente alterado aumentaram logo na primeira sessão e se equipararam ou ultrapassaram levemente as taxas nesse componente nas últimas sessões da fase Pré-Punição. As taxas de respostas no componente estável acompanharam esse aumento inicial das taxas no componente alterado na primeira sessão da fase Pós-Punição para oito dos sujeitos (PC2, PC3, PC8, PE2, PE3, PE4, PE7 e PE8), enquanto que para o restante permaneceram no patamar em que estavam nas últimas sessões da fase Punição.

A Tabela 1 mostra a taxa média de reforços, calculada com base nas seis últimas sessões de estabilidade de cada fase, para cada sujeito, nos componentes claro e escuro.
Para todos os sujeitos as taxas de reforços diminuíram (com a redução na taxa de respostas), quando o JAQ foi administrado no componente claro ou escuro (taxas $\leq$ ou $=0,50$ ), na fase Punição. Nessa fase, as taxas de reforços aumentaram no componente não alterado, para maior parte dos sujeitos (PC3, PC4, PC5, PC6, PC7, PC8, PE1, PE3, PE4, PE5 e PE7), em comparação com a fase PréPunição. Todavia, não é seguro afirmar que esse aumento tenha sido diretamente relacionado com a produção de contraste. Embora o maior aumento observado na taxa de reforços $(19 \%)$ tenha ocorrido para PE1, que foi o sujeito que apresentou contraste de forma mais expressiva, os sujeitos PC5 e PE7 não mostraram contraste e apresentaram aumentos maiores (13\%) do que outros sujeitos que apresentaram contraste (PC3 e PC8 - 6\% de aumento).
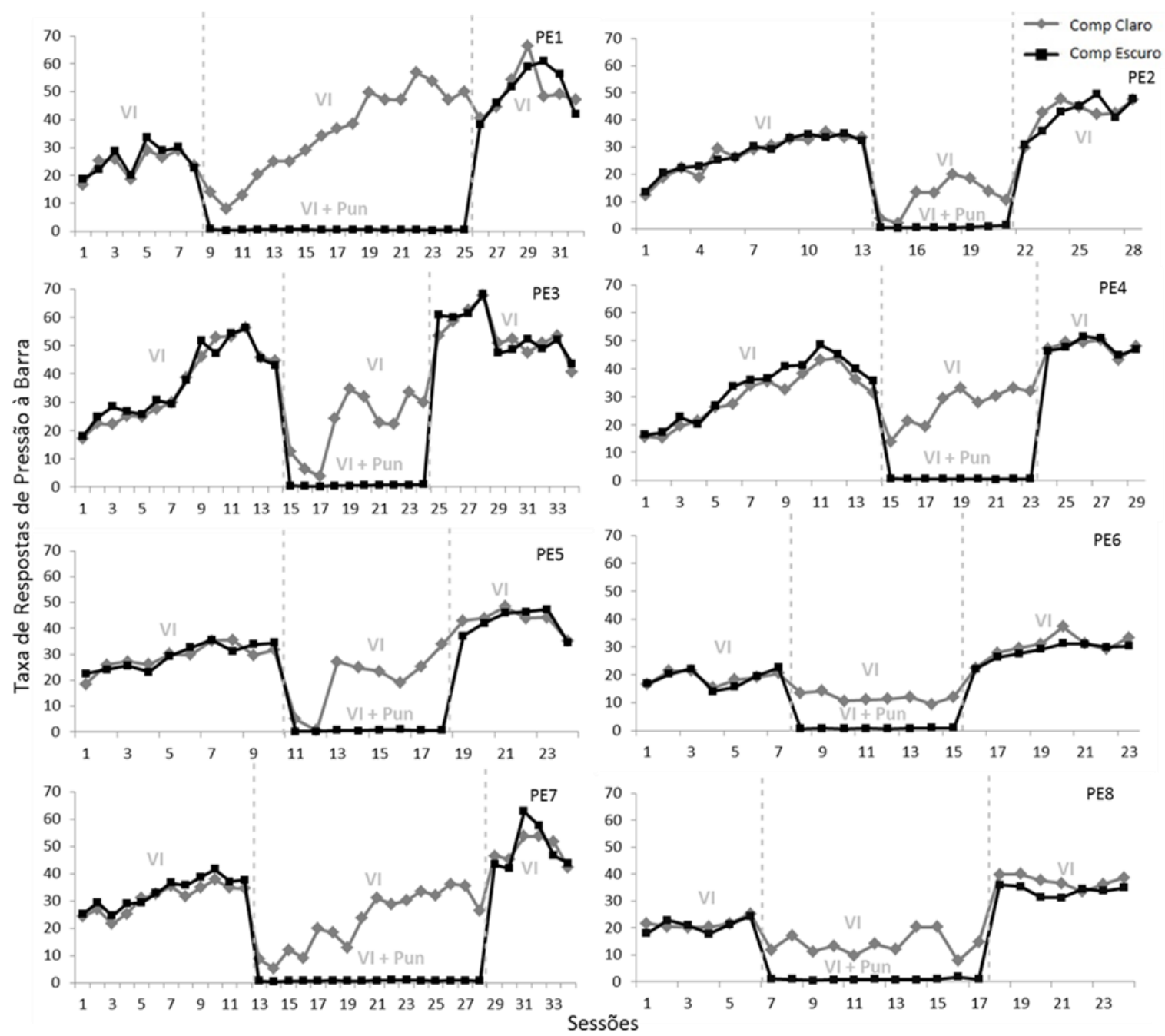

Figura 3. Taxa de respostas de pressão à barra em todas as sessões das fases Pré-Punição, Punição e Pós-Punição, quando o JAQ foi aplicado no componente escuro (mudanças de fases representadas por linhas verticais tracejadas). A linha cinza representa a taxa de respostas no componente claro e a linha preta representa a taxa de resposta no componente escuro. 
A Figura 4 mostra a diferença (em porcentagem) da taxa de respostas no componente não punido durante a fase Punição, em relação à média da taxa durante a fase PréPunição. A diferença em porcentagem no componente estável foi negativa para todos os sujeitos na primeira sessão da fase Punição, ou seja, ocorreu decréscimo acentuado no total de respostas nesse componente em comparação com as três últimas sessões da fase Pré-Punição. Essa mudança foi mais acentuada para PC2, PE2 e PE5, e menos para PC8 e PE6. Embora a diferença porcentagem tenha aumentado no decorrer das sessões seguintes, ela se manteve abaixo de 0 para a maior parte dos sujeitos, indicando que o total de respostas ficou abaixo do total médio nas três últimas sessões da fase Pré-Punição. São exceções os sujeitos PC3, PC8 e PE1, cuja diferença na porcentagem aumentou acima de zero.

Destaca-se o sujeito PE1, cuja porcentagem de mudança aumentou de forma significativa atingindo mais de $100 \%$, o que indica que, para este sujeito, o total de respostas no componente não punido, a partir da sétima sessão, ficou muito acima do total de respostas neste componente nas três últimas sessões da fase Pré-Punição. Essa análise permitiu identificar a ocorrência inicial de indução negativa para todos os sujeitos, e a ocorrência tardia de contraste positivo para três sujeitos.

\section{DISCUSSÃO}

Os dados obtidos demonstraram decréscimo na taxa de respostas no componente estável, quando o JAQ foi introduzido contingente à resposta de pressão à barra no componente alterado (claro ou escuro), para a maior parte dos sujeitos. Pode-se afirmar que a apresentação do JAQ contingente à resposta de pressão à barra funcionou como um estímulo punidor, diminuindo a taxa de respostas no componente alterado e gerando também indução negativa no componente estável, principalmente durante a primeira sessão em que o JAQ foi introduzido. Entretanto, houve recuperação de respostas no componente estável ao longo das sessões da fase Punição, para todos os sujeitos, sendo que para PC3, PC8 e PE1, a taxa atingiu valores mais altos do que na fase Pré-Punição, isto é, ocorreu contraste positivo tardio, após recuperação da indução negativa. Portanto, notase que a supressão das respostas em um componente do esquema múltiplo foi acompanhada por indução e contraste no outro componente. Considerando esse aspecto, os dados produzidos estão de acordo com os dados de Azrin e Holz (1966), Brethower e Reynolds (1962) e Crosbie et al. (1997), que também produziram indução e contraste com não humanos, utilizando choque elétrico.

Tabela 1

Taxa de Reforço (Ref/mim) em Cada Componente (Claro e Escuro), nas Três Fases Experimentais, por Sujeito.

\begin{tabular}{lcccccc}
\hline & \multicolumn{7}{c}{ Fases } \\
\hline \multirow{2}{*}{ Sujeitos } & \multicolumn{2}{c}{ Pré-Punição } & \multicolumn{2}{c}{ Punição } & \multicolumn{2}{c}{ Pós-Punição } \\
\cline { 2 - 7 } PC1 & Claro & Escuro & Claro & Escuro & Claro & Escuro \\
PC2 & 1,68 & 1,92 & $\mathbf{0 , 3 2}$ & 1,91 & 1,88 & 1,89 \\
PC3 & 1,89 & 1,91 & $\mathbf{0 , 3 0}$ & 1,89 & 1,77 & 2,02 \\
PC4 & 1,82 & 1,95 & $\mathbf{0 , 3 5}$ & 2,07 & 1,87 & 1,91 \\
PC5 & 1,78 & 1,91 & $\mathbf{0 , 3 8}$ & 2,04 & 1,83 & 1,98 \\
PC6 & 1,99 & 1,79 & $\mathbf{0 , 2 8}$ & 2,04 & 1,78 & 1,98 \\
PC7 & 1,69 & 1,90 & $\mathbf{0 , 3 8}$ & 1,98 & 1,83 & 1,93 \\
PC8 & 1,83 & 1,88 & $\mathbf{0 , 5 0}$ & 1,95 & 1,89 & 1,88 \\
& 1,73 & 1,92 & $\mathbf{0 , 3 3}$ & 2,05 & 1,91 & 1,82 \\
PE1 & 1,71 & 2,01 & 2,05 & $\mathbf{0 , 2 7}$ & 1,88 & 1,89 \\
PE2 & 1,86 & 1,88 & 1,62 & $\mathbf{0 , 1 7}$ & 1,84 & 1,98 \\
PE3 & 1,85 & 1,95 & 1,92 & $\mathbf{0 , 4 1}$ & 1,96 & 1,83 \\
PE4 & 1,82 & 1,96 & 1,93 & $\mathbf{0 , 3 6}$ & 1,86 & 1,92 \\
PE5 & 1,78 & 1,96 & 1,84 & $\mathbf{0 , 3 9}$ & 1,85 & 1,97 \\
PE6 & 1,88 & 1,68 & 1,55 & $\mathbf{0 , 4 6}$ & 1,87 & 1,89 \\
PE7 & 1,74 & 2,03 & 1,97 & $\mathbf{0 , 3 8}$ & 1,86 & 1,93 \\
PE8 & 1,87 & 1,69 & 1,63 & $\mathbf{0 , 4 3}$ & 2,01 & 1,71 \\
\hline
\end{tabular}

Nota: Os números em negrito correspondem à taxa de reforço no componente punido. 


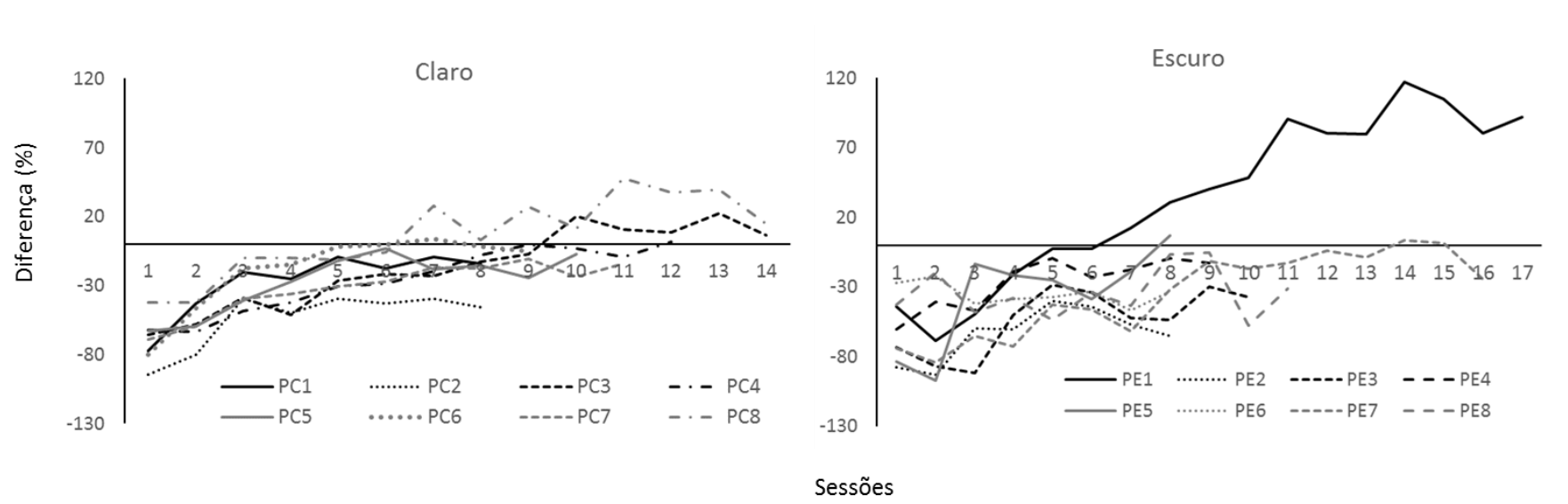

Figura 4. Diferença, em porcentagem, no total de respostas emitidas no componente não alterado na Fase Punição, em comparação com as três últimas sessões da Fase Pré-Punição. O painel à esquerda mostra os dados dos sujeitos cuja punição foi administrada no componente claro, e o da direita no componente escuro.

Todavia algumas diferenças podem ser notadas. Brethower e Reynolds (1962) produziram majoritariamente contraste comportamental, e o dado principal desse experimento foi indução. Os autores observaram que a produção de contraste estava correlacionada com a intensidade do choque, ou seja, quanto maior a intensidade do choque, maior contraste no componente estável. Essa relação não foi avaliada neste experimento, pois a intensidade do JAQ permaneceu constante.

Apenas para um dos sujeitos do experimento de Brethower e Reynolds (1962), a primeira introdução do choque elétrico produziu indução negativa, mas com repetidas exposições ao choque, o responder aumentou e houve produção de contraste (como o observado com três sujeitos do presente estudo). Brethower e Reynolds afirmam que o aparecimento de contraste pode depender de repetidas exposições à punição.

Observando os dados de PC3, PC8 e PE1, nota-se que eles foram os sujeitos que permaneceram mais tempo na condição de punição por conta do critério de estabilidade $(14,14$ e 17 sessões, respectivamente) e que, contraste foi produzido de forma visível para PE1 que teve maior número de sessões. Sendo assim, pode ser que os demais sujeitos não tenham apresentado esse dado, pelo fato de terem atingido o critério de estabilidade em poucas sessões. Os sujeitos PE7 e PC4 que também permaneceram por mais tempo na fase Punição (16 e 12 sessões, respectivamente), atingiram, nas últimas sessões, porcentagem de mudança ligeiramente acima de zero.

Sendo assim, sugere-se que em investigações futuras o critério de Schoenfeld, Cumming e Hearst (1956) seja utilizado, mas que a coleta de dados se mantenha até que o último sujeito alcance estabilidade. Dessa forma, o critério de estabilidade é garantido para todos os sujeitos, e há a possibilidade de comparação do número de sessões, para verificar se, de fato, um número maior de sessões favorece a produção de contraste comportamental.
Crosbie et al. (1997) produziram contraste comportamental para três sujeitos na primeira condição de introdução da punição e indução na segunda. Os dados produzidos por este experimento são contrários a esse achado, pois houve a produção de indução na primeira condição de introdução da punição, e contraste ocorreu posteriormente para três sujeitos. Crosbie et al. tiveram como cuidado experimental a manutenção da taxa de reforços estável, para que não houvesse covariação com os efeitos da punição.

No presente experimento essa manipulação não foi feita. Identificou-se que o aumento na taxa de reforços no componente estável não foi condição suficiente para a produção de contraste comportamental na fase Punição, pois para alguns sujeitos a taxa de reforços aumentou e ainda assim ocorreu contraste, mas também para outros sujeitos a taxa aumentou e não ocorreu contraste. Todavia, é importante salientar que o sujeito cuja ocorrência de contraste foi mais evidente (PE1) teve o maior aumento na taxa de reforços em comparação com os demais.

Sendo assim, o papel da taxa de reforços não foi claro e essa variável pode ter se misturado com o número de sessões de exposição ao punidor, apontada por Brethower e Reynolds (1962), como importante para a produção de contraste. Sugere-se que essa limitação seja eliminada em estudos futuros, mantendo-se a taxa de reforços estável.

Outro fator elucidado por Azrin e Holz (1966) e Gutman, Suttere e Brush (1975) diz respeito aos estímulos exteroceptivos correlacionados com cada componente do esquema múltiplo. Alguns estímulos não têm distinção física conspícua, como é o caso da luz e ausência de luz o que, por sua vez, pode facilitar a produção de indução. $O$ presente experimento utilizou esses estímulos correlacionados com cada componente do múltiplo, logo sugere-se que em próximos experimentos sejam utilizados estímulos com dimensões físicas mais discrepantes, por exemplo, luz e som. 
Conclui-se que o presente experimento contribuiu para aumentar a generalidade dos dados sobre interações comportamentais com punição, ao demonstrar a produção de indução e, de forma mais modesta, contraste, utilizando um punidor alternativo ao choque elétrico. Além disso, o experimento foi mais uma demonstração do JAQ como um punidor efetivo para respostas de pressão à barra em ratos.

Os resultados dão sustentação a um conjunto de dados que mostra ocorrência de indução e contraste, quando punição é introduzida em um dos componentes do esquema múltiplo. As variáveis responsáveis pela produção de um ou outro tipo de interação, bem como sua ordem de ocorrência não ficaram claras. Todavia, o presente experimento, apesar das limitações indicadas, forneceu alternativas de manipulação de variáveis que podem contribuir na identificação das condições necessárias e suficientes para a produção de indução e/ou contraste comportamental.

\section{REFERÊNCIAS}

Azrin, N. (1956). Some effects of two intermittent schedules of immediate and non-immediate punishment. The Journal of Psychology, 42, 3-21.

Azrin, N. H., \& Holz, W. C. (1966). Punishment. In W. K. Honig (Ed.), Operant behavior: Areas of research and application (pp. 380-447). New York: AppletonCentury-Crofts.

Baron, A. (1991). Avoidance and punishment. In I. H. Iversen, \& K.A. Lattal (Eds.), Experimental analysis of behavior - Part I (pp. 173-217). Amsterdan: Elsevier.

Bloomfield, T. M. (1967). Behavioral contrast and relative reinforcement frequency in two multiple schedules. Journal of the Experimental Analysis of Behavior, 10(2), 151-158. doi: 10.1901/jeab.1967.10-151.

Bradshaw, C. M. (1975). Behavioral contrast in albino rats. Psychological Reports, 37(1), 287-291. doi: 10.2466/pr0.1975.37.1.287.

Brethower, D. M., \& Reynolds, G. S. (1962). A facilitative effect of punishment on unpunished behavior. Journal of the Experimental Analysis of Behavior, 5(2), 191-199. doi: 10.1901/jeab.1962.5-191.

Caplan, M., \& Graefe, J. (1980). Punishment-induced suppression and the occurrence of behavioral contrast in rats with septal lesions. Behavioral and Neural Biology, 30(3), 278-279. doi:10.1016/S0163-1047(80)91162-0.

Carvalho Neto, M. B., Costa, J. R., Barros, R. S., Farias, D. C., \& Rico, V. V. (2013). Discriminação com três diferentes contingências em $\mathrm{S} \Delta$ : Extinção, reforçamento e punição, extinção e punição. Interação em Psicologia, 17(2), 171-179.

Carvalho Neto, M. B., Maestri, T. C., \& Menezes, E. S. R. (2007). O jato de ar quente como estímulo aversivo: Efeitos supressivos da exposição prolongada em Rattus norvegicus. Acta Comportamentalia, 15(2), 171-190.

Carvalho Neto, M. B., Maestri, T. C., Tobias, G. K. S., Ribeiro, T. C., Coutinho, E. C. N. N., Miccione, M. M., Oliveira, R. C. V., Ferreira, F. S. S., Farias, D. C., \&
Moreira, D. (2005). O jato de ar quente como estímulo punidor em Rattus norvegicus. Psicologia: Teoria e Pesquisa, 21(3), 335-339.

Carvalho Neto, M. B., Neves Filho, H. B., Borges, R. P., \& Tobias, G. K. S. (2007). Efeito da apresentação contingente (FI 1min) e não contingente (FT 1min) de um evento aversivo (jato de ar quente) sobre a frequência de pressão à barra em Rattus norvegicus. In: W. C. M. P. Silva (Org.), Sobre Comportamento e Cognição (pp. 149-153). Santo André (SP): ESETec.

Carvalho Neto, M. B., Rico, V. V., Tobias, G. K. S., Gouveia Jr, A., \& Angerami, J. G. T. (2005). O jato de ar quente como estímulo aversivo: efeitos da sua apresentação contingente e não-contingente. Em: H. J. Guilhardi \& N. C. de Aguirre. (Org.), Sobre Comportamento e Cognição (Vol. 15, pp. 400-408). Santo André (SP): ESETec.

Catania, C. A. (1961). Behavioral contrast in a multiple and concurrent schedule of reinforcement. Journal of the Experimental Analysis of Behavior, 4(4), 335-342. doi: 10.1901/jeab.1961.4-335.

Catania, C. A. (2008). The Journal of the Experimental Analysis at zero, fifty, and one hundred. Journal of the Experimental Analysis of Behavior, 89(1), 11-118. doi: 10.1901/jeab.2008.89-111.

Catania, A. C., \& Gill, C. A. (1964). Inhibition and behavioral contrast. Psychonomic Science, 1(1), 257-258. doi: 10.3758/BF03342897.

Coates, T. J. (1972). The differential effects of punishment and extinction on behavioral contrast. Psychonomic Science, 27(3), 146-148. doi: 10.3758/BF03328918.

Costa, C. E., \& Cançado, C. R. X. (2012). Stability check: A program for calculating the stability of behavior. Mexican Journal of Behavior Analysis, 38(1), 61-71. Disponível em: http://www.scielo.org.mx/pdf/rmac/v38n1/v38n1a4.pdf.

Crosbie, J., Williams, A. M., Lattal, K. A., Anderson, M. M., \& Brown, S. (1997). Schedule interactions involving punishment with pigeons and humans. Journal of the Experimental Analysis of Behavior, 68(2), 161-175. doi: 10.1901/jeab.1997.68-161.

Dinsmoor, J. A. (1952). A discrimination based on punishment. The Quarterly Journal of Experimental Psychology, 4(1), 27-45. doi: 10.1080/17470215208416601.

Fleshler, M., \& Hoffman, H. S. (1962). A progression for generating variable-interval schedules. Journal of the Experimental Analysis of Behavior, 5(4), 529-530. doi: 10.1901/jeab.1962.5-529.

Gutman, A. (1977). Positive contrast, negative induction, and inhibitory stimulus control in the rat. Journal of the Experimental Analysis of Behavior, 27(2), 219-233. doi: 10.1901/jeab.1977.27-219.

Gutman, A., Sutterer, J. R., \& Brush, F. R. (1975). Positive and negative behavioral contrast in the rat. Journal of the 
Experimental Analysis of Behavior, 23(3), 377-383. doi: 10.1901/jeab.1975.23-377.

Hemmes, N. S., \& Eckerman, D. A. (1972). Positive interaction (induction) in multiple variable-interval, differential-reinforcement-of-high-rate schedules. Journal of the Experimental Analysis of Behavior, 17(1), 51-57. doi: 10.1901/jeab.1972.17-51.

Herrnstein, R. J., \& Brady, J. V. (1958). Interaction among components of a multiple schedule. Journal of the Experimental Analysis of Behavior, 1, 293-300. doi: 10.1901/jeab.1958.1-293.

Hoffman, H., Hunt, P., \& Spear, N. E. (1991). Ontogenetic differences in CS palatability following conditioned taste aversion learning. Learning and Motivation, 22(3), 329352. Disponível em: http://dx.doi.org/10.1016/00239690(91)90012-W.

Honig, W. K., \& Slivka, R. M. (1964). Stimulus generalization of the effects of punishment. Journal of the Experimental Analysis of Behavior, 7(1), 21-25. doi: 10.1901/jeab.1964.7-21.

Hurtado-Parrado, C. (2015). Aversive control of Betta splendens behavior using water disturbances: effects of signaled and unsignalled free-operant avoidance, escape, and punishment contingencies. (Tese de Doutorado). Universidade de Manitoba, Winnipeg, MB.

Keller, F. S. (1941). Light-aversion in the white rat. Psychological Record, 4 (1), 233-249.

Keller, J. V. (1966). Delayed escape from light by albino rat. Journal of the Experimental Analysis of Behavior, 9 (6), 655- 658. doi: 10.1901/jeab.1966.9-655.

Lattal, K. A. (1970). Relative frequency of reinforcement and rate of punished behavior. Journal of the Experimental Analysis of Behavior, 13(3), 319-324. doi: 10.1901/jeab.1970.13-319.

Lattal, K. A., \& Griffin, M. A. (1972). Punishment during free-operant avoidance. Journal of the Experimental Analysis of Behavior, 18(3), 509-516. doi: 10.1901/jeab.1972.18-509.

MacDonall, J. S., \& Marcucella, H. (1978). Stimulus similarity and positive induction. Bulletin of the Psychonomic Society, 12(1), 43-46. doi: 10.3758/BF03329619.

MacSweeney, F. K., \& Norman, W. D. (1979). Defining behavioral contrast for multiple schedules. Journal of the Experimental Analysis of Behavior, 32(3), 457-461. doi: 10.1901/jeab.1979.32-457.

Mayer, P. C. M., Silva, G. F., \& Carvalho Neto, M. B. (2015). Punishment of instrumental and consummatory responses with a hot air blast in rats. Behavior Analysis: Research and Practice, 15(1), 58-64. doi: http://dx.doi.org/10.1037/h0101070.

Nascimento, G. S., \& Carvalho Neto, M. B. (2011). Supressão condicionada com diferentes estímulos aversivos: choque elétrico e jato de ar quente. Acta Comportamentalia, 19(3), 269-280. Disponível em: http://pepsic.bvsalud.org/pdf/actac/v19n3/a02.pdf.
Pear, J. J., \& Wilkie, D. (1971). Contrast and induction in rats on multiple schedules. Journal of the Experimental Analysis of Behavior, 15(3), 289-296. doi: 10.1901/jeab.1971.15-289.

Pelchat, M. L., Grill, H. J., Rozin, P., \& Jacobs, J. (1983). Quality acquired responses to tastes by Rattus norvegicus depends on type of associated discomfort. Journal of Comparative Psychology, 97(2), p. 140-153. Dísponivel em: http://dx.doi.org/10.1037/0735-7036.97.2.140.

Rachlin, H. (1966). Recovery of responses during mild punishment. Journal of the Experimental Analysis of Behavior, 9(3), 251-263. doi: 10.1901/jeab.1966.9-251.

Reynolds, G. S. (1961a). Behavioral contrast. Journal of the Experimental Analysis of Behavior, 4, 57-71. doi: 10.1901/jeab.1961.4-57.

Reynolds, G. S. (1961b). Relativity of response rate and reinforcement frequency in multiple schedule. Journal of the Experimental Analysis of Behavior, 4, 179-184. doi: 10.1901/jeab.1961.4-179.

Reynolds, G. S. (1961c). An analysis of interaction in a multiple schedule. Journal of the Experimental Analysis of Behavior, 4, 107-117. doi: 10.1901/jeab.1961.4-107.

Reynolds, G. S., \& Limpo, A. J. (1968). On some causes of behavioral contrast. Journal of the Experimental Behavior Analysis of Behavior, 11, 543-547. doi: 10.1901/jeab.1968.11-543.

Rodrigues, B. D., Nascimento, G. S., Cavalcante. L. C., \& Carvalho Neto, M. B. (2008). Efeitos da punição de uma classe de respostas usando diferentes dimensões e intensidades do jato de ar quente. Revista Brasileira de Análise do Comportamento, 4(2), 231-242.

Schoenfeld, W. N., Cumming, W. W., \& Hearst, E. (1956). On the classification of reinforcement schedules. Proceedings of the National Academy of Sciences of the United States of America, 42 (8), 563-570.

Silva, G. F., Carvalho Neto, M. B., \& Mayer, P. C. M. (2014). O jato de ar quente como estímulo aversivo antecedente. Acta Comportamentalia, 22(2), p. 135-151. Disponível em: http://pepsic.bvsalud.org/scielo.php?script=sci_arttext\&p id $=$ S0188-81452014000200002\&lng=pt\&tlng=pt.

Spealman, R. D. (1978). Interactions in multiple schedules: Negative induction in squirrel monkeys. Journal of the Experimental Analysis of Behavior, 30(3), 315-327. doi: 10.1901/jeab.1978.30-315.

Swindell, S., McSeewney, K. F., \& Murphy, E. R. (2003). Dynamic changes in the size of behavioral contrast. The Behavior Analyst Today, 4(2), 202-211. Disponível em: http://dx.doi.org/10.1037/h0100118.

Thomas, G. V., \& Cameron, G. N. (1974). Response rate, reinforcement frequency, and behavioral contrast. Journal of the Experimental Analysis of Behavior, 22(10), 427-432. doi: 10.1901/jeab.1967.10-151.

Topping, J. S., \& Larmi Jr, O. K. L. (1972). Behavioral contrast as a function of changes in reinforcement 
B. C. dos Santos, H. Q. PuReza, \& M. B. Carvalho-Neto

frequency. Psychological Reports, 30(2), 583-587.doi: 10.2466/pr0.1972.30.2.583.

Tullis, C., \& Walters, G. (1968). Punished and unpunished responding in multiple variable-interval schedules. Journal of the Experimental Analysis of Behavior, 11(2), 147-152. doi: 10.1901/jeab.1968.11-14.

Submetido em 05/09/2016

Aceito em 12/04/2017 